Commentary/Evans \& Levinson: The myth of language universals

\title{
The myth of language universals and the myth of universal grammar
}

doi:10.1017/S0140525X09990641

\author{
Morten H. Christiansen ${ }^{\mathrm{a}}$ and Nick Chater ${ }^{\mathrm{b}}$ \\ ${ }^{a}$ Department of Psychology, Cornell University, Ithaca, NY 14853, and Santa \\ Fe Institute, Santa Fe, NM 87501; ${ }^{\mathrm{b}}$ Division of Psychology and Language \\ Sciences, University College London, London, WC1E 6BT, United Kingdom. \\ christiansen@cornell.edu \\ http://www.psych.cornell.edu/people/Faculty/mhc27.htm \\ n.chater@ucl.ac.uk \\ http://www.psychol.ucl.ac.uk/people/profiles/chater_nick.htm
}

\begin{abstract}
Evans \& Levinson (E\&L) argue that language universals are a myth. Christiansen and Chater (2008) have recently suggested that innate universal grammar is also a myth. This commentary explores the connection between these two theses, and draws wider implications for the cognitive science of language.
\end{abstract}

It has been widely argued that an innate Universal Grammar (UG) must be postulated to explain two key observations: first, that languages share putatively "universal" patterns, which appear arbitrary from a functional, communicative point of view; and second, that children acquire language so readily from an apparently impoverished linguistic input (the "poverty of the stimulus" argument).

The second point has been the subject of considerable recent interest, with many theorists arguing that linguistic input is richer than has previously been suspected (e.g., Pullum \& Scholz 2002; Reali \& Christiansen 2005) or that modern learning methods are richer than is often presumed (e.g., Chater \& Vitányi 2007; Harman \& Kulkarni 2007). The first argument, based on language universals, has gone relatively unchallenged in the cognitive science literature - but no longer. Evans \& Levinson (E\&L) provide powerful evidence that language universals are myth rather than reality, and hence, that this line of defense of UG is swept aside. It remains to be explained, though, how languages came to display such stunning diversity, and this is where research on language evolution may offer some insight.

We have recently argued (Christiansen \& Chater 2008) that an innate UG is not merely poorly evidenced, but indefensible on evolutionary grounds. Specifically, we argue that the cultural variability of language provides a "moving target," which changes too rapidly to support the biological adaptation that would be required to lead to an innate UG (Chater et al. 2009). Thus, language is best viewed as the product of cultural evolution, not biological evolution (Christiansen et al., in press).

The cultural evolution of language does not, of course, take place in a biological or social vacuum, but rather, is shaped by multiple constraints. One type of constraint derives from the nature of the thoughts that language expresses. For example, whatever the nature of our mental representations, they apparently afford an infinite range of different thoughts, promoting the likely emergence of compositionality in language (Kirby 2007). Linguistic structure is also shaped by socio-pragmatic principles relating to the communicative function of language; 
for example, as embodied by Gricean implicatures (Grice 1967). A further source of constraints on language evolution derives from the operation of our perceptuo-motor apparatus, which, for example, enforces substantial seriality in both spoken and signed languages. Similarly, cognitive limitations on learning, processing, and memory also provide strong constraints on linguistic structure; for example, our limited working memory promotes a general tendency to resolve ambiguities as quickly as possible in both linguistic (Clark 1975) and perceptual input (Pomerantz \& Kubovy 1986).

Individual languages are seen as evolving under the pressures from these constraints, as well as cultural-historical factors (including language contact and sociolinguistic influences), resulting over time in the kind of linguistic diversity described by E\&L. Cross-linguistically recurring patterns do emerge due to similarity in constraints and culture/history, but such patterns should be expected to be probabilistic tendencies, not the rigid properties of UG (Christiansen \& Chater 2008). Thus, we construe recurring patterns of language along the lines of Wittgenstein's (1953) notion of "family resemblance": although there may be similarities between pairs of individual languages, there is no single set of features common to all languages.

This perspective on language evolution and universals has important implications for language acquisition and processing. The ready learnability of language is explicable not because language fits an innate $\mathrm{UG}$ within each child; but rather, because language itself embodies patterns that are most naturally acquired from past generations of learners. We have argued, more generally, that learning cultural forms (C-induction) is very much easier than learning aspects of the natural world (N-induction) - because learning merely requires that each new generations agrees with the previous generation. For example, the number sequence $1,2,3 \ldots$ could be continued in any numbers of ways using repetition $(1,2,3,1,2,3,1 \ldots)$, oscillation $(1,2,3,2,1,2 \ldots)$, as a Fibonacci sequence $(1,2,3,5,8 \ldots)$, or some entirely irregular pattern $(1,2,3,73,0,18 \ldots)$. In the context of $\mathrm{N}$-induction, the learner faces real difficulties: In encountering the sequence in some aspect of the natural world (e.g., the number of planets observed on successive nights), it is very difficult to know how the sequence will continue. However, Cinduction is much more reliable and straightforward. The vast majority of people would find it most natural to continue the sequence with “...4,5,6..." Thus, predicting how other people will continue the sequence is relatively easy, at least if people have the same inductive biases. Similarly, children must extrapolate a language from the sample of language they encounter; but such extrapolation is likely to be correct, given that it is the result of prior extrapolations by previous generations of learners. Again, the learning problem is dramatically easier if the objective is to mirror a cultural form that has been learned by others. Indeed, through prior generations of cultural selection, the form itself will have been optimized to embody whatever inductive biases the learner may have (Chater \& Christiansen, in press).

Our emphasis on C-induction in language acquisition dovetails with a usage-based approach to language processing. Connectionist cognitive science has for some time explored the computational implications of a usage-based approach to language in which constituency and recursion are not built into the architecture of the language system but rather emerge through learning as probabilistic generalizations (Christiansen \& Chater 2003). Importantly, we have developed usage-based models of recursive sentence processing that are capable of constituent-like generalization and have quasi-recursive abilities comparable to human performance on a variety of complex recursive constructions (Christiansen \& Chater 1999; Christiansen \& MacDonald, in press). Thus, at least some aspects of cognitive science do fit with the picture of language outlined by E\&L, in which recursion and constituency are not innately defined universals.
E\&L's important paper will substantially shift the debate in the cognitive science of language. Cognitive scientists have often taken rigid language universals as a "given," to be explained by theories of language acquisition and processing (e.g., by the postulation of an innate UG). E\&L make clear that this is a mistake - and that a much more nuanced view of the patterns in the world's languages is required. We argue that this perspective is consistent with the view that an innate UG is as much a myth as language universals; and that language should be viewed as primarily a product of cultural, rather than biological, evolution. 